\title{
On commutators of certain fractional type integrals with Lipschitz functions
}

Wenting $\mathrm{Hu}^{1}$, Yongming Wen ${ }^{1}$ and Huoxiong $\mathrm{Wu}^{1 *}$

${ }^{\text {"Correspondence: }}$

huoxwu@xmu.edu.cn

'School of Mathematical Sciences,

Xiamen University, Xiamen, China

\begin{abstract}
In this paper, we study the commutators generated by Lipschitz functions and fractional type integral operators with kernels of the form

$$
K_{\alpha}(x, y)=\kappa_{1}\left(x-A_{1} y\right) \kappa_{2}\left(x-A_{2} y\right) \cdots \kappa_{m}\left(x-A_{m} y\right)
$$

where $0 \leq \alpha=\alpha_{1}+\cdots+\alpha_{m}<n$, each $\kappa_{i}$ satisfies the $\left(n-\alpha_{i}\right)$-order fractional size condition and a generalized fractional Hörmander condition, $A_{i}$ is invertible, and $A_{i}-A_{j}$ is invertible for $i \neq j, 1 \leq i, j \leq m$. We establish the corresponding sharp maximal function estimates and obtain the weighted Coifman type inequalities, weighted $L^{p}\left(W^{P}\right) \rightarrow L^{q}\left(W^{q}\right)$ estimates, and the weighted endpoint estimates for such commutators.
\end{abstract}

MSC: Primary 42B25; secondary 42B20

Keywords: Commutators; Lipschitz functions; Generalized fractional Hörmander condition; Fractional size condition; Weighted estimates; Coifman type inequality

\section{Introduction and main results}

Let $n, m \in \mathbb{N}, 0 \leq \alpha<n$. For any locally integrable bounded function $f$, define

$$
T_{\alpha, m} f(x):=\int_{\mathbb{R}^{n}} K_{\alpha}(x, y) f(y) d y,
$$

where

$$
K_{\alpha}(x, y)=k_{1}\left(x-A_{1} y\right) k_{2}\left(x-A_{2} y\right) \cdots k_{m}\left(x-A_{m} y\right),
$$

$\alpha=\alpha_{1}+\cdots+\alpha_{m}$, and for each $1 \leq i \leq m, k_{i}$ satisfies $\left(n-\alpha_{i}\right)$-order fractional size condition, $A_{i}$ is a matrix such that

(H) $A_{i}$ is invertible and $A_{i}-A_{j}$ is invertible for $i \neq j, 1 \leq i, j \leq m$.

Clearly, $T_{\alpha, 1}=I_{\alpha}$, the Riesz potential, for $m=1, A_{1}$ is the $n$-order identity matrix, and $k_{1}\left(x-A_{1} y\right)=1 /|x-y|^{\alpha}$. For general $m$ and certain $k_{i}, T_{0, m}$ behaves like a singular integral operator and $T_{\alpha, m}$ has been studied in [1-10]. In particular, Riveros and Urciuolo [5, 6, 11] considered each $k_{i}$ as a rough fractional kernel, and each $k_{i}$ satisfies an $L^{\alpha_{i}, \gamma_{i}}$-Hörmander

(c) The Author(s) 2019. This article is distributed under the terms of the Creative Commons Attribution 4.0 International License (http://creativecommons.org/licenses/by/4.0/), which permits unrestricted use, distribution, and reproduction in any medium, provided you give appropriate credit to the original author(s) and the source, provide a link to the Creative Commons license, and indicate if changes were made. 
regular condition, or more general $k_{i} \in H_{\alpha, \gamma_{i}}$, that is, for all $x \in \mathbb{R}^{n}$ and $|x|<R$,

$$
\sum_{j=1}^{\infty}\left(2^{j} R\right)^{n-\alpha}\left\|\left(K_{\alpha}(\cdot-x)-K_{\alpha}(\cdot)\right) \chi_{B\left(x, 2^{j+1} R\right) \backslash B\left(x, 2^{j} R\right)}\right\|_{\gamma_{i}, B\left(x, 2^{m} R\right)}<\infty
$$

They showed that these operators are bounded from $L^{p}$ into $L^{q}$, for $1<p \leq q<\infty, 1 / q=$ $1 / p-\alpha / n$. In [12], Ibañez-Firnkorn and Riveros analyzed operators of the form (1) with conditions of regularity more general than the $L^{\alpha, \gamma}$-Hörmander condition and a fractional size condition. Before giving the definitions of the fractional size condition $S_{n-\alpha_{i}, \Psi_{i}}$ and the generalized fractional Hörmander condition $H_{n-\alpha_{i}, \psi_{i}, k}$, we first recall the definitions and properties for Young function.

A function $\Psi:[0, \infty) \rightarrow[0, \infty)$ is said to be a Young function if $\Psi$ is continuous, convex, nondecreasing and satisfies $\Psi(0)=0$ and $\lim _{t \rightarrow \infty} \Psi(t)=\infty$. For $f \in L_{\mathrm{loc}}^{1}\left(\mathbb{R}^{n}\right)$ and each Young function $\Psi$, we can induce an average of the Luxemburg norm of a function $f$ in the ball $B$ defined by

$$
\|f\|_{\Psi, B}:=\inf \left\{\lambda>0: \frac{1}{|B|} \int_{B} \Psi\left(\frac{|f(x)|}{\lambda}\right) d x \leq 1\right\},
$$

and a fractional maximal operator $M_{\alpha, \Psi}(0 \leq \alpha<n)$ defined by

$$
M_{\alpha, \Psi} f(x):=\sup _{B \ni x}|B|^{\alpha / n}\|f\|_{\Psi, B}
$$

and we denote $M_{0, \Psi}$ by $M_{\Psi}$, the Orlicz maximal operator.

In particular, for $\Psi(t)=t,\|f\|_{\Psi, B}:=|B|^{-1} \int_{B}|f(x)| d x$ and $M_{\alpha, \Psi}=M_{\alpha}$, the fractional maximal operator; for $\Psi(t)=t^{r}$ with $1<r<\infty,\|f\|_{\Psi, B}=\|f\|_{r, B}:=\left(|B|^{-1} \int_{B}|f(x)|^{r} d x\right)^{1 / r}$ and $M_{\alpha, \Psi}=M_{\alpha, r}$, and $M_{0, r} f:=\sup _{B \ni x}\|f\|_{r, B}:=M\left(f^{r}\right)^{1 / r}$.

Next, we recall the definitions of the fractional size condition and the generalized fractional Hörmander condition. Normally, we use $|x| \sim s$ to represent $s<|x| \leq 2 s$. For the Young function $\Psi$, we write

$$
\|f\|_{\Psi,|x| \sim s}=\left\|f \chi_{|x| \sim s}\right\|_{\Psi, B(0,2 s)} .
$$

For $0 \leq \alpha<n$, the function $K_{\alpha}$ is said to satisfy the fractional size condition if there exists a constant $C>0$ such that

$$
\left\|K_{\alpha}\right\|_{\Psi,|x| \sim s} \leq C s^{\alpha-n} .
$$

And we denote $K_{\alpha} \in S_{\alpha, \Psi}$ in this case. When $\Psi(t)=t$, we write $S_{\alpha, \Psi}=S_{\alpha}$. Observe that if $K_{\alpha} \in S_{\alpha}$, then there exists a constant $C>0$ such that

$$
\int_{|x| \sim s}\left|K_{\alpha}(x)\right| d x \leq C s^{\alpha} .
$$

We say that the function $K_{\alpha}$ satisfies the $L^{\alpha, \Psi, k}$-Hörmander condition denoted by $K_{\alpha} \in$ $H_{\alpha, \Psi, k}$ if there exist constants $c_{\Psi}>1$ and $C_{\Psi}>0$ such that, for all $x$ and $R>c_{\Psi}|x|$,

$$
\sum_{j=1}^{\infty}\left(2^{j} R\right)^{n-\alpha} j^{k}\left\|K_{\alpha}(\cdot-x)-K_{\alpha}(\cdot)\right\|_{\Psi,|y| \sim 2 j R} \leq C_{\Psi} .
$$


When $\Psi(t)=t^{r}, 1 \leq r<\infty$, we simply write $H_{\alpha, r, k}$ instead of $H_{\alpha, \Psi, k}$. See [13] or [14] for more details.

In this paper, we consider the $k$-order commutators $T_{\alpha, m, b}^{k}$ generated by Lipschitz functions and the operator $T_{\alpha, m}$, where $k_{i} \in S_{n-\alpha_{i}, \Psi_{i}} \cap H_{n-\alpha_{i}, \Psi_{i}, k}$, and for $k \in \mathbb{N} \cup\{0\}$,

$$
T_{\alpha, m, b}^{k}(f)(x)=\int_{\mathbb{R}^{n}}(b(x)-b(y))^{k} K_{\alpha}(x, y) f(y) d y .
$$

Clearly, $T_{\alpha, m, b}^{0}=T_{\alpha, m}$.

Also, we consider the following condition for the weights: there exists $C>0$ such that

$$
\omega\left(A_{i} x\right) \leq C \omega(x), \quad \text { a.e. } x \in \mathbb{R}^{n}
$$

for all $1 \leq i \leq m$. Let $\omega=\left\{\begin{array}{ll}\log \frac{1}{|x|}, & |x| \leq e^{-1}, \\ 1, & |x|>e^{-1}\end{array}\right.$. It is easy to check that $\omega \in A_{1}$ and satisfies (2) (see [15]).

In [14], Gallo, Ibañez-Firnkorn, and Riveros obtained the weighted estimates for this kind of operator and certain weights satisfying (2). Precisely as for the classical fractional integral operator $I_{\alpha}$ with $0<\alpha<n$, or the singular integral operator with $\alpha=0$, they proved the $L^{p}\left(\mathbb{R}^{n}, \omega^{p}\right) \rightarrow L^{q}\left(\mathbb{R}^{n}, \omega^{q}\right)$ boundedness of $T_{\alpha, m}$ for weights $\omega \in A(p, q), 1<p<n / \alpha$, $1 / q=1 / p-\alpha / n$, and $0 \leq \alpha<n$. In [15], for $b \in$ BMO, Ibañez-Firnkorn and Riveros obtained the weighted Coifman type estimates, weighted $L^{p}\left(\omega^{p}\right) \rightarrow L^{q}\left(\omega^{q}\right)$ estimates, and weighted BMO estimates as well as two-weighted inequalities. Inspired by these results, we consider the weighted boundedness of $T_{\alpha, m, b}^{k}$ for $b \in \dot{\Lambda}_{\beta}$ and a weighted $\dot{\Lambda}_{\beta}$ estimate for weights in the class $A(n /(\alpha+k \beta) r, \infty)$. Our results can be formulated as follows.

Theorem 1.1 For $0<\beta<1,0 \leq \alpha<n, k \in \mathbb{N} \cup\{0\}, m \in \mathbb{N}$, and $1 \leq i \leq m$, let $b \in \dot{\Lambda}_{\beta}, \Psi_{i}$ be Young functions and $0 \leq \alpha_{i}<n$ such that $\alpha_{1}+\cdots+\alpha_{n}=n-\alpha$. Let $T_{\alpha, m}$ be the integral operator defined by (1) and $T_{\alpha, m, b}^{k}$ be the k-order commutator of $T_{\alpha, m}$. Suppose that the matrices $A_{i}$ satisfy hypothesis $(\mathrm{H})$ and $k_{i} \in S_{n-\alpha_{i}, \Psi_{i}} \cap H_{n-\alpha_{i}, \Psi_{i}, k}$. Moreover, for $\alpha=0$, suppose that $T_{0, m}$ is strong type $\left(q_{*}, q_{*}\right)$ for some $1<q_{*}<\infty$. Let $\varphi_{k}(t)=t \log (e+t)^{k}, \phi$ be a Young function satisfying $\Psi_{1}^{-1}(t) \cdots \Psi_{m}^{-1}(t) \phi^{-1}(t){\overline{\varphi_{k}}}^{-1}(t) \lesssim t$ for $t \geq t_{0}$, some $t_{0}>0$. Then there exists $0<C=C\left(n, \alpha, A_{1}, \ldots, A_{m}\right)$ such that, for $0<\delta \leq 1$ and $f \in L_{c}^{\infty}\left(\mathbb{R}^{n}\right)$,

$$
M_{\delta}^{\sharp}\left(T_{\alpha, m, b}^{k} f\right)(x) \leq C \sum_{l=0}^{k-1}\|b\|_{\dot{A}_{\beta}}^{k-l} M_{(k-l) \beta}\left(T_{\alpha, m, b}^{l} f\right)(x)+C\|b\|_{\dot{A}_{\beta}}^{k} \sum_{i=0}^{m} M_{\alpha+k \beta, \phi} f\left(A_{i}^{-1} x\right) .
$$

Theorem 1.2 Under the assumptions of Theorem 1.1, for $1 \leq r<p<p_{l} \leq q<\infty, k, l \in \mathbb{N}$, $1 / q=1 / p_{l}-(k-l) \beta / n, 1 / q=1 / p-(\alpha+k \beta) / n$, there exists $0<C=C\left(n, \alpha, A_{1}, \ldots, A_{m}\right)$ such that, for $f \in L_{c}^{\infty}\left(\mathbb{R}^{n}\right)$ and $\omega^{r} \in A(p / r, q / r)$,

$$
\left\|T_{\alpha, m, b}^{k} f\right\|_{L^{q}\left(\omega^{q)}\right.} \leq C\|b\|_{\dot{\Lambda}_{\beta}}^{k} \sum_{i=1}^{m} \sum_{l=0}^{k}\left(\int_{\mathbb{R}^{n}}\left|M_{\alpha+l \beta, \phi} f(x)\right|^{p_{l}} \omega^{p_{l}}\left(A_{i} x\right) d x\right)^{1 / p_{l}} .
$$

Furthermore, if $\omega^{r} \in A(p / r, q / r)$ and satisfying (2), then

$$
\left\|T_{\alpha, m, b}^{k} f\right\|_{L^{q}\left(\omega^{q}\right)} \leq C\|b\|_{\dot{A}_{\beta}}^{k} \sum_{l=0}^{k}\left\|M_{\alpha+l \beta, \phi} f\right\|_{L^{p l}\left(\omega^{p l)}\right)}
$$


Theorem 1.3 Let $0 \leq \alpha<n, 1<p<n /(\alpha+k \beta), 1 / q=1 / p-(\alpha+k \beta) / n$ and $\phi$ be a Young function such that $\eta^{-1}(t) t^{\frac{(\alpha+k \beta)}{n}} \lesssim \phi^{-1}(t)$ for every $t>0$, where $\phi^{1+\frac{s n}{n-(\alpha+k \beta)}} \in B_{\frac{s n}{n-(\alpha+k \beta)}}$ for every $s>r(n-(\alpha+k \beta)) /(n-(\alpha+k \beta) r)$. Then, under the hypotheses of Theorem 1.2, for $\omega^{r} \in$ $A(p / r, q / r)$,

$$
\left\|T_{\alpha, m, b}^{k} f\right\|_{L^{q}\left(\omega^{q)}\right)} \leq C\|b\|_{\dot{\Lambda}_{\beta}}^{k}\|f\|_{L^{p}\left(\omega^{p}\right)}
$$

Theorem 1.4 Under the hypotheses of Theorem 1.3, if $\omega^{r} \in A(n /(\alpha+k \beta) r, \infty)$ and satisfies (2), then there exists $C>0$ such that, for $f \in L_{c}^{\infty}\left(R^{n}\right)$,

$$
\left\|T_{\alpha, m, b}^{k} f \mid\right\|_{\omega} \leq C\|b\|_{\dot{\Lambda}_{\beta}}^{k}\|f \omega\|_{L^{n / \alpha+k \beta}},
$$

where

$$
\left\|\left|T_{\alpha, m, b}^{k} f\right|\right\|_{\omega}=\sup _{B}\left\|\omega \chi_{B}\right\|_{\infty}\left(\frac{1}{|B|} \int_{B}\left|T_{\alpha, m, b}^{k} f(y)-\frac{1}{|B|} \int_{B} T_{\alpha, m, b}^{k} f(z) d z\right| d y\right) .
$$

The rest of this paper is organized as follows. In Sect. 2 we recall some relevant definitions and previous results that are needed to state the other results, which appear in Sect. 1. The proofs of sharp maximal functions estimates and Coifman type inequalities are given in Sect. 3. Finally, the weighted $L^{p}\left(w^{p}\right) \rightarrow L^{q}\left(w^{q}\right)$ estimates and the weighted endpoint estimates are presented in Sect. 4.

\section{Preliminaries}

In this section we present some relevant concepts and previous results, which will be used in our proofs.

\subsection{The generalized Hölder inequality and the fractional $B_{p}$ condition}

Now, we present some extra properties for Young functions. For more details of these topics, see [16] or [17].

The function $\bar{\Psi}$ is called the complementary of the function $\Psi$ if the generalized Hölder inequality holds:

$$
\|f g\|_{L_{1}, B} \leq 2\|f\|_{\Psi, B}\|g\|_{\bar{\Psi}, B} .
$$

If $\Psi_{1}, \ldots, \Psi_{m}, \phi$ are Young functions satisfying $\Psi_{1}^{-1}(t) \cdots \Psi_{m}^{-1}(t) \phi^{-1}(t) \lesssim t$ for $t \geq t_{0}$, some $t_{0}>0$, then

$$
\left\|f_{1} \cdots f_{m} g\right\|_{L_{1}, B} \leq c\left\|f_{1}\right\|_{\Psi_{1}, B} \cdots\left\|f_{m}\right\|_{\Psi_{m}, B}\|g\|_{\phi, B}
$$

where the function $\phi$ is called the complementary of the functions $\Psi_{1}, \ldots, \Psi_{m}$.

In 2013, Cruz-Uribe and Moen [18] introduced the fractional $B_{p}$ condition: for $1<p<$ $n / \alpha$ and $1 / q=1 / p-\alpha / n$, a Young function $\phi \in B_{p}^{\alpha}$ if

$$
\int_{1}^{\infty} \frac{\phi(t)^{q / p}}{t^{q}} \frac{d t}{t}<\infty
$$


And they proved that if $\phi \in B_{p}^{\alpha}$, then $M_{\alpha, \phi}: L^{p}(d x) \rightarrow L^{q}(d x)$ and

$$
\left\|M_{\alpha, \phi}\right\|_{L^{p} \rightarrow L^{q}} \leq\left(\int_{1}^{\infty} \frac{\phi(t)^{q / p}}{t^{q}} \frac{d t}{t}\right)^{1 / q}
$$

\subsection{The Lipschitz function spaces}

For a locally integrable function $f$ defined in $\mathbb{R}^{n}$, we say $f$ belongs to the space $\dot{\Lambda}_{\beta}\left(\mathbb{R}^{n}\right)$, $0<\beta<1$, if there exists a constant $C>0$ such that

$$
\sup _{B \ni x} \frac{1}{|B|^{1+\beta / n}} \int_{B}\left|f(x)-f_{B}\right| d x<\infty
$$

The smallest bound $C$ satisfying upper inequality is taken to be the norm of $f$ denoted by $\|f\|_{\dot{\Lambda}_{\beta}}$. Here $B$ is a ball in $\mathbb{R}^{n}$, and

$$
f_{B}=\frac{1}{|B|} \int_{B} f(x) d x
$$

Lemma 2.1 If $\in \in \dot{\Lambda}_{\beta}$, then

(1) for every $x, y \in \mathbb{R}^{n}$,

$$
|f(x)-f(y)| \leq\|f\|_{\dot{\Lambda}_{\beta}}|x-y|^{\beta}
$$

(2) for any ball $B$,

$$
\sup _{x \in B}\left|f(x)-f_{B}\right| \leq C\|f\|_{\dot{A}_{\beta}}|B|^{\beta / n}
$$

(3) for $B \subset B^{*}$,

$$
\left|f_{B}-f_{B^{*}}\right| \leq\|f\|_{\dot{\Lambda}_{\beta}}\left|B^{*}\right|^{\beta / n} .
$$

In particular, if $A_{i}$ are matrices satisfying $(\mathrm{H}), \tilde{B}$ is a measurable set, and $\tilde{B}_{i}=A_{i}^{-1} \tilde{B}, 1 \leq i \leq m$, then

$$
\left|f_{\tilde{B}}-f_{\left(\bigcup_{i=1}^{m} \tilde{B}_{i}\right) \cup \tilde{B}}\right| \leq C\|f\|_{\dot{A}_{\beta}}|\tilde{B}|^{\beta / n},
$$

and for $B=B\left(c_{B}, R\right)$, the ball centered at $c_{B}$ with radius $R$, and $B^{j}:=B\left(c_{B}, 2^{j} R\right), j \in \mathbb{N}$,

$$
\left|f_{B}-f_{B^{j}}\right| \leq C j\|f\|_{\dot{A}_{\beta}}\left|B^{j}\right|^{\beta / n}
$$

\subsection{Weights and maximal operators}

A weight function $\omega$ is in the Muckenhoupt class $A_{p}$ for $1<p<\infty$ if there exists $C>1$ such that, for any ball $B$,

$$
\left(\frac{1}{|B|} \int_{B} \omega(x) d x\right)\left(\frac{1}{|B|} \int_{B} \omega(x)^{1-p^{\prime}} d x\right)^{p-1} \leq C
$$


where $1 / p+1 / p^{\prime}=1$ and the infimum of $C$ satisfying the above inequality is denoted by $[\omega]_{A_{p}}$. We define $A_{\infty}=\bigcup_{1 \leq p<\infty} A_{p}$. When $p=1, \omega \in A_{1}$ if there exists $C>1$ such that, for almost every $x$,

$$
M \omega(x) \leq C \omega(x)
$$

and the infimum of $C$ satisfying the above inequality is denoted by $[\omega]_{A_{1}}$.

A weight function $\omega$ belongs to $A(p, q)$ for $1<p<q<\infty$ if there exists $C>1$ such that

$$
\left(\frac{1}{|B|} \int_{B} \omega(x)^{q} d x\right)^{1 / q}\left(\frac{1}{|B|} \int_{B} \omega(x)^{-p^{\prime}} d x\right)^{1 / p^{\prime}} \leq C
$$

where $1 / p+1 / p^{\prime}=1$ and the infimum of $C$ satisfying the above inequality is denoted by $[\omega]_{A_{p, q}}$. When $p=1, \omega$ is in $A(1, q)$ with $1<q<\infty$ if there exists $C>1$ such that

$$
\left(\frac{1}{|B|} \int_{B} \omega(x)^{q} d x\right)\left(\operatorname{ess} \sup _{x \in B} \frac{1}{\omega(x)}\right) \leq C
$$

and the infimum of $C$ satisfying the above inequality is denoted by $[\omega]_{A_{1, q}}$.

Remark 2.1 For $1 \leq r<p<p_{0} \leq p, \omega \in A(p, q)$, by Hölder's inequality, we can know $\omega \in$ $A\left(p_{0}, q\right)$ and $\omega \in A\left(p, p_{0}\right)$. For $\omega^{r} \in A(p / r, q / r)$, we also can know $\omega \in A(p, q)$.

The sharp maximal function is defined by

$$
M^{\sharp} f(x)=\sup _{B \ni x} \frac{1}{|B|} \int_{B}\left|f(y)-\frac{1}{|B|} \int_{B} f(z) d z\right| d y .
$$

A locally integrable function $f$ has bounded mean oscillation $(f \in \mathrm{BMO})$ if $M^{\sharp} f(x) \in L^{\infty}$ and the norm $\|f\|_{\text {BMO }}=\left\|M^{\sharp} f\right\|_{\infty}$. Observe that the BMO norm is equivalent to

$$
\|f\|_{\mathrm{BMO}}=\left\|M^{\sharp} f\right\|_{\infty} \sim \sup _{B} \inf _{a \in \mathbb{C}} \frac{1}{|B|} \int_{B}|f(y)-a| d y .
$$

There is also a weighted version of $\mathrm{BMO}$, which is denoted by $\mathrm{BMO}(\omega)$, which is described by the semi-norm

$$
\|f\|_{\omega}=\sup _{B}\left\|\omega \chi_{B}\right\|_{\infty}\left(\frac{1}{|B|} \int_{B}\left|f(y)-\frac{1}{|B|} \int_{B} f(z) d z\right| d y\right) .
$$

It is easy to check that

$$
\|f\|_{\omega} \simeq\left\|\omega M^{\sharp} f\right\|_{\infty} .
$$

Proposition 2.1 ([19]) Let $\Psi$ be a Young function. Then, for all $x \in \mathbb{R}^{n}$ and $r>1$, there exists a constant $C_{r}$ such that

$$
M_{\Psi} f(x)=M_{0, \Psi} f(x) \leq C_{r} M_{0, r} f(x) .
$$


Proposition 2.2 ([15]) Let $\Psi$ be a Young function and $A$ be an invertible matrix. Set $\omega_{A}(x)=\omega(A x)$. Then

$$
M_{\alpha, \Psi}\left(\omega_{A}\right)\left(A^{-1} x\right) \leq c_{A, n} M_{\alpha, \Psi}(\omega)(x)
$$

for almost every $x \in \mathbb{R}^{n}$.

\subsection{Previous results}

In this subsection, we illustrate some known results for the operator $T_{\alpha, m}$, which will be used below, see [12] for more details.

Theorem 2.1 ([12]) Let $0 \leq \alpha<n, m \in \mathbb{N}$, and $T_{\alpha, m}$ be the integral operator defined by (1). For $1 \leq i \leq m$, let $\Psi_{i}$ be Young functions, $0 \leq \alpha_{i}<n$ such that $\alpha_{1}+\cdots+\alpha_{m}=n-\alpha$. Also suppose $k_{i} \in S_{n-\alpha_{i}, \Psi_{i}} \cap H_{n-\alpha_{i}, \psi_{i}}$ and that matrices $A_{i}$ satisfy hypothesis $(\mathrm{H})$.

If $\alpha=0$, suppose $T_{0, m}$ is of strong type $\left(q_{*}, q_{*}\right)$ for some $1<q_{*}<\infty$.

If $\phi$ is the complementary of the functions $\Psi_{1}, \ldots, \Psi_{m}$, then there exists $C>0$ such that, for $0<\delta \leq 1$ and $f \in L_{c}^{\infty}\left(\mathbb{R}^{n}\right)$,

$$
M_{\delta}^{\sharp}\left(T_{\alpha, m} f\right)(x):=M^{\sharp}\left(\left|T_{\alpha, m} f\right|^{\delta}\right)(x)^{1 / \delta} \leq C \sum_{i=1}^{m} M_{\alpha, \phi} f\left(A_{i}^{-1} x\right) .
$$

Theorem 2.2 ([12]) Let $0 \leq \alpha<n, m \in \mathbb{N}$, and $T_{\alpha, m}$ be the integral operator defined by (1). For $1 \leq i \leq m$, let $\Psi_{i}$ be Young functions, $0 \leq \alpha_{i}<n$ such that $\alpha_{1}+\cdots+\alpha_{m}=n-\alpha$. Also suppose $k_{i} \in S_{n-\alpha_{i}, \psi_{i}} \cap H_{n-\alpha_{i}, \psi_{i}}$ and that matrices $A_{i}$ satisfy hypothesis $(\mathrm{H})$.

When $\alpha=0$, suppose that $T_{0, m}$ is of strong type $\left(q_{*}, q_{*}\right)$ for some $1<q_{*}<\infty$.

Let $0<p<\infty$. If $\phi$ is the complementary of the functions $\Psi_{1}, \ldots, \Psi_{m}$, then there exists $C>0$ such that, for $\omega \in A_{\infty}$ and $f \in L_{c}^{\infty}\left(\mathbb{R}^{n}\right)$,

$$
\int_{\mathbb{R}^{n}}\left|T_{\alpha, m} f(x)\right|^{p} \omega(x) d x \leq C \sum_{i=1}^{m} \int_{\mathbb{R}^{n}}\left|M_{\alpha, \phi} f(x)\right|^{p} \omega\left(A_{i} x\right) d x,
$$

whenever the left-hand side is finite.

\section{Sharp maximal function estimates and Coifman type inequality}

This section is devoted to the proofs of Theorems 1.1 and 1.2.

Proof of Theorem 1.1 We just consider the case $m=2$ and $k=1$, i.e., $T_{\alpha, 2, b}^{1}=\left[b, T_{\alpha, 2}\right]$, and we will just write $\left[b, T_{\alpha}\right]$ for simplicity. The general case is proved in an analogous way.

Let $f$ be a bounded function with compact support, $0<\delta \leq 1$. For $x \in \mathbb{R}^{n}$, let $B=B\left(c_{B}, R\right)$ be a ball that contains $x$, centered at $c_{B}$ with radius $R$. We write $\tilde{B}=B\left(c_{B}, 2 R\right)$, and for $1 \leq i \leq 2$, set $\tilde{B}_{i}=A_{i}^{-1} \tilde{B}$. Let $f_{1}=f \chi_{\bigcup_{i=1}^{2} \tilde{B}_{i}}$ and $f_{2}=f-f_{1}$. Suppose that $a:=T_{\alpha}\left(\left(b_{\tilde{B} \cup \tilde{B}_{1} \cup \tilde{B}_{2}}-\right.\right.$ b) $f)\left(c_{B}\right)<\infty$. For $0<\delta \leq 1$, we write

$$
\left[b, T_{\alpha}\right](f)(x)=\left(b(x)-b_{\tilde{B} \cup \tilde{B}_{1} \cup \tilde{B}_{2}}\right) T_{\alpha} f(x)+T_{\alpha}\left(\left(b_{\tilde{B} \cup \tilde{B}_{1} \cup \tilde{B}_{2}}-b\right) f\right)(x) .
$$

And from the inequality $\left|t^{\delta}-s^{\delta}\right|^{1 / \delta} \leq|t-s|$ and Jensen's inequality, we get

$$
\left(\frac{1}{|B|} \int_{B}\left|\left[b, T_{\alpha}\right](f)^{\delta}(y)-a^{\delta}\right| d y\right)^{1 / \delta}
$$




$$
\begin{aligned}
\leq & \left(\frac{1}{|B|} \int_{B}\left|\left[b, T_{\alpha}\right](f)(y)-a\right|^{\delta} d y\right)^{1 / \delta} \\
\leq & \frac{1}{|B|} \int_{B}\left|\left(b(y)-b_{\tilde{B} \cup \tilde{B}_{1} \cup \tilde{B}_{2}}\right) T_{\alpha} f(y)\right| d y \\
& +\frac{1}{|B|} \int_{B}\left|T_{\alpha}\left(\left(b_{\tilde{B} \cup \tilde{B}_{1} \cup \tilde{B}_{2}}-b\right) f_{1}\right)(y)\right| d y \\
& +\frac{1}{|B|} \int_{B}\left|T_{\alpha}\left(\left(b_{\tilde{B} \cup \tilde{B}_{1} \cup \tilde{B}_{2}}-b\right) f_{2}\right)(y)-T_{\alpha}\left(\left(b_{\tilde{B} \cup \tilde{B}_{1} \cup \tilde{B}_{2}}-b\right) f_{2}\right)\left(c_{B}\right)\right| d y \\
= & I+I I+I I I .
\end{aligned}
$$

For $I$, by Lemma 2.1, we have

$$
\begin{aligned}
I & \leq C\|b\|_{{\dot{A}}_{\beta}}|B|^{\beta / n}\left(\frac{1}{|B|} \int_{B}\left|T_{\alpha} f(y)\right| d y\right) \\
& \leq C\|b\|_{{\dot{\Lambda_{\beta}}}_{\beta}} M_{\beta}\left(T_{\alpha} f\right)(x) .
\end{aligned}
$$

For $I I$, we know

$$
\begin{aligned}
I I & =\frac{1}{|B|} \int_{B} \int_{\tilde{B}_{1} \cup \tilde{B}_{2}}\left|K_{\alpha}(y, z)\right|\left|b(z)-b_{\tilde{B} \cup \tilde{B}_{1} \cup \tilde{B}_{2}}\right|\left|f_{1}(z)\right| d z d y \\
& \leq \sum_{i=1}^{2} \frac{1}{|B|} \int_{\tilde{B}_{i}}\left|b(z)-b_{\tilde{B}^{\prime} \cup \tilde{B}_{1} \cup \tilde{B}_{2}}\right|\left|f_{1}(z)\right| \int_{B}|K(y, z)| d y d z .
\end{aligned}
$$

We estimate only the first summand, that is, $z \in \tilde{B}_{1}$, since the case $z \in \tilde{B}_{2}$ is analogous. Observe that

$$
\int_{B}\left|K_{\alpha}(y, z)\right| d y \leq \int_{\left\{y \in B:\left|y-A_{1} z\right| \leq\left|y-A_{2} z\right|\right\}}\left|K_{\alpha}(y, z)\right| d y+\int_{\left\{y \in B:\left|y-A_{2} z\right| \leq\left|y-A_{1} z\right|\right\}}\left|K_{\alpha}(y, z)\right| d y .
$$

For $j \in \mathbb{N}$, let us consider the set

$$
C_{j}^{1}:=\left\{y \in B:\left|y-A_{1} z\right| \leq\left|y-A_{2} z\right|,\left|y-A_{1} z\right| \sim 2^{-j-1} R\right\} .
$$

Notice that if $y \in B$ and $z \in \tilde{B}_{1}$, then $\left|y-A_{1} z\right| \leq 3 R<4 R$. Thus,

$$
\begin{aligned}
& \int_{\left\{y \in B:\left|y-A_{1} z\right| \leq\left|y-A_{2} z\right|\right\}}\left|K_{\alpha}(y, z)\right| d y \\
& \leq \sum_{j=-2}^{\infty} \int_{C_{j}^{1}}\left|K_{\alpha}(y, z)\right| d y \\
& \leq \sum_{j=-2}^{\infty} \frac{\left|B\left(A_{1} z, 2^{-j} R\right)\right|}{\left|B\left(A_{1} z, 2^{-j} R\right)\right|} \int_{B\left(A_{1} z 2^{-j} R\right)}\left|K_{\alpha}(y, z)\right| \chi_{\left\{y:\left|y-A_{1} z\right| \sim 2^{-j-1} R\right\}} d y \\
& \leq C \sum_{j=-2}^{\infty}\left|B\left(A_{1} z, 2^{-j} R\right)\right|\left\|k_{1}\left(\cdot-A_{1} z\right) \chi_{\left\{y:\left|y-A_{1} z\right| \sim 2^{-j-1} R\right\}}\right\|_{\Psi_{1}, B\left(A_{1} z, 2^{-j} R\right)} \\
& \quad \times\left\|k_{2}\left(\cdot-A_{2} z\right) \chi_{\left\{y:\left|y-A_{1} z\right| \sim 2^{-j-1} R\right\}}\right\|_{\Psi_{2}, B\left(A_{1} z, 2^{-j} R\right)}
\end{aligned}
$$




$$
\begin{aligned}
\leq & C \sum_{j=-2}^{\infty}\left|B\left(A_{1} z, 2^{-j} R\right)\right|\left\|k_{1}\left(\cdot-A_{1} z\right)\right\|_{\Psi_{1},\left|y-A_{1} z\right| \sim 2^{-j-1} R} \\
& \times\left\|k_{2}\left(\cdot-A_{2} z\right)\right\|_{\Psi_{2},\left|y-A_{1} z\right| \sim 2^{-j-1} R^{.}}
\end{aligned}
$$

And for $y \in C_{j}^{1}$, we have $\left|y-A_{2} z\right| \geq\left|y-A_{1} z\right| \geq 2^{-j-1} R$. By $k_{2} \in S_{n-\alpha_{2}, \Psi_{2}}$ and $k_{1} \in S_{n-\alpha_{1}, \Psi_{1}}$, we get

$$
\begin{aligned}
\left\|k_{2}\left(\cdot-A_{2} z\right)\right\|_{\Psi_{2},\left|y-A_{1} z\right| \sim 2^{-j-1} R} & \leq \sum_{k \geq 0}\left\|k_{2}\left(\cdot-A_{2} z\right)\right\|_{\Psi_{2},\left|y-A_{2} z\right| \sim 2^{-j+k-1} R} \\
& \leq \sum_{k \geq 0}\left\|k_{2}(\cdot)\right\|_{\Psi_{2},|y| \sim 2^{-j+k-1} R} \\
& \leq C \sum_{k \geq 0}\left(2^{-j+k-1} R\right)^{-\alpha_{2}} \leq C\left(2^{-j} R\right)^{-\alpha_{2}},
\end{aligned}
$$

and

$$
\left\|k_{1}\left(\cdot-A_{1} z\right)\right\|_{\Psi_{1},\left|y-A_{1} z\right| \sim 2^{-j-1} R} \leq C\left(2^{-j-1} R\right)^{-\alpha_{1}} \leq C\left(2^{-j} R\right)^{-\alpha_{1}} .
$$

Consequently,

$$
\int_{\left\{y \in B:\left|y-A_{1} z\right| \leq\left|y-A_{2} z\right|\right\}}\left|K_{\alpha}(y, z)\right| d y \leq C \sum_{j=-2}^{\infty}\left(2^{-j} R\right)^{n-\alpha_{1}-\alpha_{2}} \leq C R^{\alpha} .
$$

Similarly,

$$
\int_{\left\{y \in B:\left|y-A_{2} z\right| \leq\left|y-A_{1} z\right|\right\}}\left|K_{\alpha}(y, z)\right| d y \leq C R^{\alpha} .
$$

Then

$$
\begin{aligned}
I I & \leq C R^{\alpha} \sum_{i=1}^{2} \frac{1}{|B|} \int_{\tilde{B}_{i}}\left|b(z)-b_{\tilde{B} \cup \tilde{B}_{1} \cup \tilde{B}_{2}}\right|\left|f_{1}(z)\right| d z \\
& \leq C\|b\|_{\dot{A}_{\beta}} \sum_{i=1}^{2} R^{\alpha+\beta} \frac{1}{\left|\tilde{B}_{i}\right|} \int_{\tilde{B}_{i}}|f(z)| d z \\
& \leq C\|b\|_{\dot{A}_{\beta}} \sum_{i=1}^{2} R^{\alpha+\beta}\|f\|_{\phi, \tilde{B}_{i}} \\
& \leq C\|b\|_{\dot{\Lambda}_{\beta}} \sum_{i=1}^{2} M_{\alpha+\beta, \phi} f\left(A_{i}^{-1} x\right) .
\end{aligned}
$$

For III, we have

$$
\begin{aligned}
I I I & =\frac{1}{|B|} \int_{B} \int_{\left(\tilde{B}_{1} \cup \tilde{B}_{2}\right)^{c}}\left|K_{\alpha}(y, z)-K_{\alpha}\left(c_{B}, z\right)\right|\left|b(z)-b_{\tilde{B} \cup \tilde{B}_{1} \cup \tilde{B}_{2}}\right||f(z)| d z d y \\
& \leq \sum_{l=1}^{2} \frac{1}{|B|} \int_{B} \int_{Z^{l}}\left|K_{\alpha}(y, z)-K_{\alpha}\left(c_{B}, z\right)\right|\left|b(z)-b_{\tilde{B} \cup \tilde{B}_{1} \cup \tilde{B}_{2}}\right||f(z)| d z d y,
\end{aligned}
$$


where

$$
Z^{l}=\left(\tilde{B}_{1} \cup \tilde{B}_{2}\right)^{c} \cap\left\{z:\left|c_{B}-A_{l} z\right| \leq\left|c_{B}-A_{r} z\right|, r \neq l, 1 \leq r \leq 2\right\} .
$$

Let us estimate $\left|K_{\alpha}(y, z)-K_{\alpha}\left(c_{B}, z\right)\right|$ for $y \in B$ and $z \in Z^{l}$ :

$$
\begin{aligned}
\left|K_{\alpha}(y, z)-K_{\alpha}\left(c_{B}, z\right)\right| \leq & \left|k_{1}\left(y-A_{1} z\right)-k_{1}\left(c_{B}-A_{1} z\right)\right|\left|k_{2}\left(y-A_{2} z\right)\right| \\
& +\left|k_{2}\left(y-A_{2} z\right)-k_{2}\left(c_{B}-A_{2} z\right)\right|\left|k_{1}\left(c_{B}-A_{1} z\right)\right| .
\end{aligned}
$$

For simplicity we estimate the first summand of $\left|K_{\alpha}(y, z)-K_{\alpha}\left(c_{B}, z\right)\right|$, the other one follows in an analogous way. For $j \in \mathbb{N}$, let

$$
D_{j}^{l}:=\left\{z \in Z^{l}:\left|c_{B}-A_{l} z\right| \sim 2^{j+1} R\right\} .
$$

Observe that $D_{j}^{l} \subset\left\{z:\left|c_{B}-A_{l} z\right| \sim 2^{j+1} R\right\} \subset A_{l}^{-1} B\left(c_{B}, 2^{j+2} R\right)=: \tilde{B}_{l, j}$. Using the generalized Hölder inequality, we have

$$
\begin{aligned}
& \int_{Z_{l}}\left|k_{1}\left(y-A_{1} z\right)-k_{1}\left(c_{B}-A_{1} z\right)\right|\left|k_{2}\left(y-A_{2} z\right)\right|\left|b(z)-b_{\tilde{B} \cup \tilde{B}_{1} \cup \tilde{B}_{2}}\right||f(z)| d z \\
& \leq \sum_{j=1}^{\infty} \int_{D_{j}^{l}}\left|k_{1}\left(y-A_{1} z\right)-k_{1}\left(c_{B}-A_{1} z\right)\right|\left|k_{2}\left(y-A_{2} z\right)\right|\left|b(z)-b_{\tilde{B} \cup \tilde{B}_{1} \cup \tilde{B}_{2}}\right||f(z)| d z \\
& \leq \sum_{j=1}^{\infty} \frac{\left|\tilde{B}_{l, j}\right|}{\left|\tilde{B}_{l, j}\right|} \int_{\tilde{B}_{l, j}}\left|k_{1}\left(y-A_{1} z\right)-k_{1}\left(c_{B}-A_{1} z\right)\right|\left|k_{2}\left(y-A_{2} z\right)\right| \chi_{D_{j}^{l}} \chi_{\left\{z:\left|c_{B}-A_{l} z\right| \sim 2^{j+1} R\right\}} \\
& \times\left(\left|b(z)-b_{\tilde{B}_{l, j}}\right|+\left|b_{\tilde{B}_{l, j}}-b_{\tilde{B}_{l}}\right|+\left|b_{\tilde{B}_{l}}-b_{\tilde{B} \cup \tilde{B}_{1} \cup \tilde{B}_{2}}\right|\right)|f(z)| d z \\
& \leq \sum_{j=1}^{\infty} \frac{\left|\tilde{B}_{l, j}\right|}{\left|\tilde{B}_{l, j}\right|} \int_{\tilde{B}_{l, j}}\left|k_{1}\left(y-A_{1} z\right)-k_{1}\left(c_{B}-A_{1} z\right)\right|\left|k_{2}\left(y-A_{2} z\right)\right| \chi_{D_{j}^{l}} \chi_{\left\{z:\left|c_{B}-A_{l} z\right| \sim 2^{j+1} R\right\}} \\
& \times\left(C\|b\|_{\dot{\Lambda}_{\beta}}\left|\tilde{B}_{l, j}\right|^{\beta / n}+C j\|b\|_{\dot{\Lambda}_{\beta}}\left|\tilde{B}_{l, j}\right|^{\beta / n}+C\|b\|_{\dot{\Lambda}_{\beta}}|B|^{\beta / n}\right)|f(z)| d z \\
& \leq C\|b\|_{\dot{A}_{\beta}} \sum_{j=1}^{\infty}\left|\tilde{B}_{l, j}\right|^{1+\beta / n} j \frac{1}{\left|\tilde{B}_{l, j}\right|} \int_{\tilde{B}_{l, j}}\left|k_{1}\left(y-A_{1} z\right)-k_{1}\left(c_{B}-A_{1} z\right)\right| \\
& \times\left|k_{2}\left(y-A_{2} z\right)\right| \chi_{D_{j}^{l}} \chi_{\left\{z:\left|c_{B}-A_{l} z\right| \sim 2^{j+1} R_{R}\right\}}|f(z)| d z \\
& \leq C\|b\|_{\dot{A}_{\beta}} \sum_{j=1}^{\infty}\left|\tilde{B}_{l, j}\right|^{1+\beta / n} j\left\|\left(k_{1}\left(y-A_{1} \cdot\right)-k_{1}\left(c_{B}-A_{1} \cdot\right)\right) \chi_{D_{j}^{l}}\right\|_{\Psi_{1},\left|c_{B}-A_{l} z\right| \sim 2^{j+1} R} \\
& \times\left\|k_{2}\left(y-A_{2} \cdot\right) \chi_{D_{j}^{l}}\right\|_{\Psi_{2},\left|c_{B}-A_{l} z\right| \sim 2^{j+1} R}\|f\|_{\phi, \tilde{B}_{l, j}} .
\end{aligned}
$$

Note that $\left|c_{B}-A_{l} z\right| / 2 \leq\left|y-A_{l} z\right| \leq 2\left|c_{B}-A_{l} z\right|$, and if $\left|c_{B}-A_{l} z\right| \sim 2^{j+1} R$, then $2^{j} R \leq \mid y-$ $A_{l} z \mid \leq 2^{j+2} R$. Thus,

$$
\begin{gathered}
\left\|k_{l}\left(y-A_{l} \cdot\right) \chi_{D_{j}^{l}}\right\|_{\Psi_{l},\left|c_{B}-A_{l} z\right| \sim 2^{j+1} R} \\
\quad \leq\left\|k_{l}\left(y-A_{l} \cdot\right)\right\|_{\Psi_{l}\left|y-A_{l} z\right| \sim 2^{j} R}
\end{gathered}
$$




$$
\begin{aligned}
& +\left\|k_{l}\left(y-A_{l} \cdot\right)\right\|_{\Psi_{l,\left|y-A_{l} z\right| \sim 2^{j+1} R}} \\
\leq & \left\|k_{l}(\cdot)\right\|_{\Psi_{l},|x| \sim 2 j_{R}}+\left\|k_{l}(\cdot)\right\|_{\Psi_{l,|x| \sim 2^{j+1} R}} \\
\leq & C\left(2^{j} R\right)^{-\alpha_{l}},
\end{aligned}
$$

where the last inequality holds since $k_{l} \in S_{n-\alpha_{l}, \Psi_{l}}$. Also, by the hypothesis,

$$
\left\|k_{l}\left(c_{B}-A_{l} \cdot\right) \chi_{D_{j}^{l}}\right\|_{\Psi_{l, \mid c_{B}-A}-A_{l} \mid \sim 2^{j+1} R} \leq C\left(2^{j+1} R\right)^{-\alpha_{l}} .
$$

For $r \neq l$, observe that if $z \in D_{j}^{l}$, then $\left|c_{B}-A_{r} z\right| \geq\left|c_{B}-A_{l} z\right| \geq 2^{j+1} R$. We decompose $D_{j}^{l}=$ $\bigcup_{k \geq j}\left(D_{j}^{l}\right)_{k, r}$, where

$$
\left(D_{j}^{l}\right)_{k, r}=\left\{z \in D_{j}^{l}:\left|c_{B}-A_{r} z\right| \sim 2^{k+1} R\right\} .
$$

Since $\left(D_{j}^{l}\right)_{k, r} \subset\left\{z:\left|c_{B}-A_{r} z\right| \sim 2^{k+1} R\right\}$ and $k_{r} \in S_{n-\alpha_{r}, \Psi_{r}}$, we have

$$
\begin{aligned}
\left\|k_{r}\left(y-A_{r}\right) \chi_{D_{j}^{l}}\right\|_{\Psi_{r},\left|c_{B}-A_{l} z\right| \sim 2^{j+1} R} & \leq \sum_{k \geq j}\left\|k_{r}\left(y-A_{r} \cdot\right) \chi_{\left(D_{j}^{l}\right) k_{, r}}\right\|_{\Psi_{r},\left|c_{B}-A_{l} z\right| \sim 2^{j+1} R} \\
& \leq \sum_{k \geq j}\left\|k_{r}\left(y-A_{r} \cdot\right) \chi_{\left(D_{j}^{l}\right)_{k, r}}\right\|_{\Psi_{r},\left|c_{B}-A_{r} z\right| \sim 2^{k+1} R} \\
& \leq \sum_{k \geq j}\left\|k_{r}\left(y-A_{r} \cdot\right)\right\|_{\Psi_{r},\left|c_{B}-A_{r} z\right| \sim 2^{k+1} R} \\
& \leq \sum_{k \geq j}\left\|k_{r}(\cdot)\right\|_{\Psi_{r},|x| \sim 2^{k} R}+\left\|k_{r}(\cdot)\right\|_{\Psi_{r},|x| \sim 2^{k+1} R} \\
& \leq C \sum_{k \geq j}\left(2^{k} R\right)^{-\alpha_{r}} \leq C\left(2^{j} R\right)^{-\alpha_{r}} .
\end{aligned}
$$

By the same arguments, we can get

$$
\begin{aligned}
\left\|k_{r}\left(c_{B}-A_{r} \cdot\right) \chi_{D_{j}^{l}}\right\|_{\Psi_{r},\left|c_{B}-A_{|l|}\right| \sim 2^{j+1} R} & \leq \sum_{k \geq j}\left\|k_{r}\left(c_{B}-A_{r} \cdot\right) \chi_{\left(D_{j}^{l}\right)_{k, r}}\right\|_{\Psi_{r},\left|c_{B}-A_{r} z\right| \sim 2^{k+1} R} \\
& \leq C \sum_{k \geq j}\left(2^{k} R\right)^{-\alpha_{r}} \leq C\left(2^{j} R\right)^{-\alpha_{r}} .
\end{aligned}
$$

As a result, no matter $l=1$ or $l=2$, we have

$$
\begin{aligned}
& \left\|k_{2}\left(y-A_{2} \cdot\right) \chi_{D_{j}^{l}}\right\|_{\Psi_{2},\left|c_{B}-A_{l} z\right| \sim 2^{j+1} R} \leq C\left(2^{j} R\right)^{-\alpha_{2}}, \\
& \left\|k_{1}\left(c_{B}-A_{1} \cdot\right) \chi_{D_{j}^{l}}\right\|_{\Psi_{1},\left|c_{B}-A_{l} z\right| \sim 2^{j+1} R} \leq C\left(2^{j} R\right)^{-\alpha_{1}} .
\end{aligned}
$$

Hence,

$$
\begin{aligned}
& \sum_{j=1}^{\infty}\left|\tilde{B}_{l, j}\right|^{1+\beta / n} j\left\|\left(k_{1}\left(y-A_{1} \cdot\right)-k_{1}\left(c_{B}-A_{1} \cdot\right)\right) \chi_{D_{j}^{l}}\right\|_{\Psi_{1},\left|c_{B}-A_{l} z\right| \sim 2^{j+1} R} \\
& \times\left\|k_{2}\left(y-A_{2} \cdot\right) \chi_{D_{j}^{l}}\right\|_{\Psi_{2},\left|c_{B}-A_{l} z\right| \sim 2^{j+1} R}\left\|f_{2}\right\|_{\phi, \tilde{B}_{l, j}}
\end{aligned}
$$




$$
\begin{aligned}
& \leq C \sum_{j=1}^{\infty}\left(2^{j} R\right)^{n+\beta-\alpha_{2}} j\left\|\left(k_{1}\left(y-A_{1} \cdot\right)-k_{1}\left(c_{B}-A_{1} \cdot\right)\right) \chi_{D_{j}^{l}}\right\|_{\Psi_{1},\left|c_{B}-A_{l} z\right| \sim 2^{j+1} R}\|f\|_{\phi, \tilde{B}_{l, j}} \\
& =C \sum_{j=1}^{\infty}\left(2^{j} R\right)^{\alpha+\beta}\|f\|_{\phi, \tilde{B}_{l, j}}\left(2^{j} R\right)^{n-\alpha-\alpha_{2}} j\left\|\left(k_{1}\left(y-A_{1} \cdot\right)-k_{1}\left(c_{B}-A_{1} \cdot\right)\right) \chi_{D_{j}^{l}}\right\|_{\Psi_{1},\left|c_{B}-A_{l} z\right| \sim 2^{j+1} R} \\
& =C \sum_{j=1}^{\infty}\left(2^{j} R\right)^{\alpha+\beta}\|f\|_{\phi, \tilde{B}_{l, j}}\left(2^{j} R\right)^{\alpha_{1}} j\left\|\left(k_{1}\left(y-A_{1} \cdot\right)-k_{1}\left(c_{B}-A_{1} \cdot\right)\right) \chi_{D_{j}^{l} l}\right\|_{\Psi_{1},\left|c_{B}-A_{l} z\right| \sim 2^{j+1} R^{R}}
\end{aligned}
$$

So, when $l=1$, from $k_{1} \in H_{n-\alpha_{1}, \Psi_{1}, 1}$, we can get

$$
\begin{aligned}
& \sum_{j=1}^{\infty}\left(2^{j} R\right)^{\alpha+\beta}\|f\|_{\phi, \tilde{B}_{l, j}}\left(2^{j} R\right)^{\alpha_{1}} j\left\|\left(k_{1}\left(y-A_{1} \cdot\right)-k_{1}\left(c_{B}-A_{1} \cdot\right)\right) \chi_{D_{j}^{l}}\right\|_{\Psi_{1},\left|c_{B}-A_{l} z\right| \sim 2^{j+1} R} \\
& \quad \times\left\|k_{2}\left(y-A_{2} \cdot\right) \chi_{D_{j}^{l}}\right\|_{\Psi_{2},\left|c_{B}-A_{l} z\right| \sim 2^{j+1} R} \\
& \leq C M_{\alpha+\beta, \phi} f\left(A_{1}^{-1} x\right) \sum_{j=1}^{\infty}\left(2^{j} R\right)^{\alpha_{1}} j\left\|\left(k_{1}\left(y-A_{1} \cdot\right)-k_{1}\left(c_{B}-A_{1} \cdot\right)\right) \chi_{D_{j}^{l}}\right\|_{\Psi_{1},\left|c_{B}-A_{l} z\right| \sim 2^{j+1} R} \\
& \leq C M_{\alpha+\beta, \phi} f\left(A_{1}^{-1} x\right) .
\end{aligned}
$$

For $l=2$, note that

$$
\begin{aligned}
& \left\|\left(k_{1}\left(y-A_{1} \cdot\right)-k_{1}\left(c_{B}-A_{1} \cdot\right)\right) \chi_{D_{j}^{2}}\right\|_{\Psi_{1},\left|c_{B}-A_{2} z\right| \sim 2^{j+1} R} \\
& \quad \leq \sum_{k \geq j}\left\|\left(k_{1}\left(y-A_{1} \cdot\right)-k_{1}\left(c_{B}-A_{1} \cdot\right)\right) \chi_{\left.\left(D_{j}^{2}\right)\right)_{k, 1}}\right\|_{\Psi_{1},\left|c_{B}-A_{1} z\right| \sim 2^{k+1} R^{\prime}}
\end{aligned}
$$

we have

$$
\begin{aligned}
& \sum_{j=1}^{\infty}\left(2^{j} R\right)^{\alpha_{1}} j\left\|\left(k_{1}\left(y-A_{1} \cdot\right)-k_{1}\left(c_{B}-A_{1} \cdot\right)\right) \chi_{D_{j}^{2}}\right\|_{\Psi_{1},\left|c_{B}-A_{2} z\right| \sim 2^{j+1} R} \\
& \quad \leq \sum_{j=1}^{\infty}\left(2^{j} R\right)^{\alpha_{1}} j \sum_{k \geq j}\left\|\left(k_{1}\left(y-A_{1} \cdot\right)-k_{1}\left(c_{B}-A_{1} \cdot\right)\right) \chi_{\left(D_{j}^{2}\right)_{k, 1}}\right\|_{\Psi_{1},\left|c_{B}-A_{1} z\right| \sim 2^{k+1} R} \\
& \quad \leq \sum_{j=1}^{\infty}\left(2^{j} R\right)^{\alpha_{1}} \sum_{k \geq j} \frac{\left(2^{k} R\right)^{\alpha_{1}}}{\left(2^{k} R\right)^{\alpha_{1}}} k\left\|\left(k_{1}\left(y-A_{1} \cdot\right)-k_{1}\left(c_{B}-A_{1} \cdot\right)\right) \chi_{\left(D_{j}^{2}\right)_{k, 1}}\right\|_{\Psi_{1},\left|c_{B}-A_{1} z\right| 2^{k+1} R} \\
& \quad \leq \sum_{j=1}^{\infty} \sum_{k \geq j} \frac{\left(2^{j} R\right)^{\alpha_{1}}}{\left(2^{k} R\right)^{\alpha_{1}}}\left(2^{k} R\right)^{\alpha_{1}} k\left\|\left(k_{1}\left(y-A_{1} \cdot\right)-k_{1}\left(c_{B}-A_{1} \cdot\right)\right) \chi_{\left(D_{j}^{2}\right)_{k, 1}}\right\|_{\Psi_{1},\left|c_{B}-A_{1} z\right| \sim 2^{k+1} R} \\
& \quad \leq \sum_{k=1}^{\infty}\left(\sum_{j=1}^{k}\left(2^{-\alpha_{1}}\right)^{k-j}\right)\left(2^{k} R\right)^{\alpha_{1}} k\left\|\left(k_{1}\left(y-A_{1} \cdot\right)-k_{1}\left(c_{B}-A_{1} \cdot\right)\right) \chi_{\left(D_{j}^{2}\right)_{k, 1}}\right\|_{\Psi_{1},\left|c_{B}-A_{1} z\right| 2^{k+1} R} \\
& \quad \leq \sum_{k=1}^{\infty}\left(2^{k} R\right)^{\alpha_{1}} k\left\|\left(k_{1}\left(y-A_{1} \cdot\right)-k_{1}\left(c_{B}-A_{1} \cdot\right)\right) \chi_{\left(D_{j}^{2}\right)_{k, 1}}\right\|_{\Psi_{1},\left|c_{B}-A_{1} z\right| \sim 2^{k+1} R}<\infty,
\end{aligned}
$$


where the last inequality follows from that $k_{1} \in H_{n-\alpha_{1}, \Psi_{1}, 1}$. Hence,

$$
\begin{aligned}
& \sum_{j=1}^{\infty}\left(2^{j} R\right)^{\alpha+\beta}\|f\|_{\phi, \tilde{B}_{l, j}}\left(2^{j} R\right)^{\alpha_{1}} j\left\|\left(k_{1}\left(y-A_{1} \cdot\right)-k_{1}\left(c_{B}-A_{1} \cdot\right)\right) \chi_{D_{j}^{2}}\right\|_{\Psi_{1},\left|c_{B}-A_{l} z\right| \sim 2^{j+1} R} \\
& \quad \leq C M_{\alpha+\beta, \phi} f\left(A_{2}^{-1} x\right) \sum_{j=1}^{\infty}\left(2^{j} R\right)^{\alpha_{1}} j\left\|\left(k_{1}\left(y-A_{1} \cdot\right)-k_{1}\left(c_{B}-A_{1} \cdot\right)\right) \chi_{D_{j}^{2}}\right\|_{\Psi_{1},\left|c_{B}-A_{l} z\right| \sim 2^{j+1} R} \\
& \quad \leq C M_{\alpha+\beta, \phi} f\left(A_{2}^{-1} x\right) .
\end{aligned}
$$

Then

$$
I I I \leq C\|b\|_{\dot{\Lambda}_{\beta}} \sum_{i=1}^{2} M_{\alpha+\beta, \phi} f\left(A_{i}^{-1} x\right) .
$$

Summing up (4)-(7), we know that

$$
M_{\delta}^{\sharp}\left(T_{\alpha, m, b}^{1} f\right)(x) \leq C\|b\|_{\dot{\Lambda}_{\beta}} M_{\beta}\left(T_{\alpha} f\right)(x)+C\|b\|_{\dot{\Lambda}_{\beta}} \sum_{i=0}^{2} M_{\alpha+\beta, \phi} f\left(A_{i}^{-1} x\right) .
$$

For the case $\alpha=0$, we repeat the same argument to inequality (4) and get the desired conclusion.

For the general case, from the definition of $T_{\alpha, m, b}^{k}$, we know that, for any $\lambda$,

$$
\begin{aligned}
T_{\alpha, m, b}^{k}(f)(y) \\
=\int_{\mathbb{R}^{n}}(b(y)-b(z))^{k} K_{\alpha}(y, z) f(z) d z \\
=\int_{\mathbb{R}^{n}}(b(y)-\lambda+\lambda-b(z))^{k} K_{\alpha}(y, z) f(z) d z \\
=\sum_{i=0}^{k} \int_{\mathbb{R}^{n}} c_{k i}(b(y)-\lambda)^{i}(\lambda-b(z))^{k-i} K_{\alpha}(y, z) f(z) d z \\
=\sum_{i=0}^{k}(b(y)-\lambda)^{i} \int_{\mathbb{R}^{n}} c_{k i}(\lambda-b(z))^{k-i} K_{\alpha}(y, z) f(z) d z \\
=\int_{\mathbb{R}^{n}}(\lambda-b(z))^{k} K_{\alpha}(y, z) f(z) d z \\
\quad+\sum_{i=1}^{k} c_{k i}(b(y)-\lambda)^{i} \int_{\mathbb{R}^{n}}(\lambda-b(y)+b(y)-b(z))^{k-i} K_{\alpha}(y, z) f(z) d z \\
=\sum_{i=1}^{k} c_{k i}(b(y)-\lambda)^{i} \sum_{j=0}^{k-i} c_{k j}(\lambda-b(y))^{j} \int_{\mathbb{R}^{n}}(b(y)-b(z))^{k-i-j} K_{\alpha}(y, z) f(z) d z \\
\quad+T_{\alpha, m}\left((\lambda-b(\cdot))^{k} f\right)(y) \\
=\sum_{i=1}^{k} \sum_{j=0}^{k-i} c_{k i j}(b(y)-\lambda)^{i+j} \int_{\mathbb{R}^{n}}(b(y)-b(z))^{k-i-j} K_{\alpha}(y, z) f(z) d z
\end{aligned}
$$




$$
\begin{aligned}
& +T_{\alpha, m}\left((\lambda-b(\cdot))^{k} f\right)(y) \\
= & T_{\alpha, m}\left((\lambda-b(\cdot))^{k} f\right)(y)+\sum_{l=0}^{k-1} c_{k l}(b(y)-\lambda)^{k-l} T_{\alpha, m, b}^{l} f(y) .
\end{aligned}
$$

Let $\lambda:=b_{\tilde{B} \cup \tilde{B}_{1} \cup \tilde{B}_{2} \cup \ldots \cup \tilde{B}_{m}}, a:=T_{\alpha}\left(\left(b-b_{\tilde{B} \cup \tilde{B}_{1} \cup \tilde{B}_{2} \cup \ldots \cup \tilde{B}_{m}}\right) f_{2}\right)\left(c_{B}\right)$. We write

$$
\begin{aligned}
\left.\left(\frac{1}{|B|} \int_{B}\left|T_{\alpha, m, b}^{k}(f)(y)-a\right|^{\delta}\right) d y\right)^{1 / \delta} & \\
\leq & \left(\frac{1}{|B|} \int_{B}\left|\sum_{i=0}^{k-1}(b(y)-\lambda)^{k-i} T_{\alpha, m, b}^{i} f(y)\right|^{\delta} d y\right)^{1 / \delta} \\
& \left.+\left.\left(\frac{1}{|B|} \int_{B} \mid T_{\alpha}(\lambda-b(\cdot))^{k} f_{1}\right)(y)\right|^{\delta} d y\right)^{1 / \delta} \\
& \left.\left.+\left(\frac{1}{|B|} \int_{B} \mid T_{\alpha}(\lambda-b(\cdot))^{k} f_{2}\right)(y)-T_{\alpha}(\lambda-b(\cdot))^{k} f_{2}\right)\left.\left(c_{B}\right)\right|^{\delta} d y\right)^{1 / \delta} \\
= & I V+V+V I .
\end{aligned}
$$

To estimate $I V$, by Hölder's inequality and Lemma 2.1, we obtain

$$
\begin{aligned}
I V & \leq \sum_{l=0}^{k-1}\left(\frac{1}{|B|} \int_{B}\left|(b(y)-\lambda)^{k-l} T_{\alpha, m, b}^{l} f(y)\right|^{\delta} d y\right)^{1 / \delta} \\
& \leq \sum_{l=0}^{k-1} C\|b\|_{\dot{\Lambda}_{\beta}}^{k-l}|B|^{(k-l) \beta / n}\left(\frac{1}{|B|} \int_{B}\left|T_{\alpha, m, b}^{l} f(y)\right|^{\delta} d y\right)^{1 / \delta} \\
& \leq \sum_{l=0}^{k-1} C\|b\|_{\dot{\Lambda}_{\beta}}^{k-l}|B|^{(k-l) \beta / n} \frac{1}{|B|} \int_{B}\left|T_{\alpha, m, b}^{l} f(y)\right| d y \\
& \leq C \sum_{l=0}^{k-1}\|b\|_{\dot{\Lambda}_{\beta}}^{k-l} M_{(k-l) \beta}\left(T_{\alpha, m, b}^{l} f\right)(x) .
\end{aligned}
$$

The terms $V$ and $V I$ are analogous to the ones in the case $m=2$ and $k=1$, we can get

$$
\begin{aligned}
& V \leq C\|b\|_{\dot{\Lambda}_{\beta}}^{k} \sum_{i=1}^{m} M_{\alpha+k \beta, \phi} f\left(A_{i}^{-1} x\right), \\
& V I \leq C\|b\|_{\dot{\Lambda}_{\beta}}^{k} \sum_{i=1}^{m} M_{\alpha+k \beta, \phi} f\left(A_{i}^{-1} x\right) .
\end{aligned}
$$

Then we conclude

$$
M_{\delta}^{\sharp}\left|T_{\alpha, m, b}^{k} f\right|(x) \leq C \sum_{l=0}^{k-1}\|b\|_{\dot{\Lambda}_{\beta}}^{k-l} M_{(k-l) \beta}\left(T_{\alpha, m, b}^{l} f\right)(x)+C\|b\|_{\dot{\Lambda}_{\beta}}^{k} \sum_{i=1}^{m} M_{\alpha+k \beta, \phi} f\left(A_{i}^{-1} x\right) .
$$

Theorem 1.1 is proved.

Next, we prove Theorem 1.2. 
Proof of Theorem 1.2 By the extrapolation result Theorem 1.1 in [20], we need only to show that (3) is true for some $0<q_{*}<\infty$ and all $\omega^{r} \in A\left(p / r, q_{*} / r\right)$ with $(n-\alpha) / n<q_{*}<\infty$. Without loss of generality, we may assume $\|b\|_{\dot{\Lambda}_{\beta}}=1$. We will prove the desired conclusion by induction.

When $k=0, T_{\alpha, m, b}^{0}=T_{\alpha, m}$. As $k_{i} \in H_{n-\alpha_{i}, \Psi_{i}, 0}=H_{n-\alpha_{i}, \Psi_{i}}$, Theorem 3.3 in [15] tells us that

$$
\int_{\mathbb{R}^{n}}\left|T_{\alpha, m} f(x)\right|^{q_{*}} \omega^{q_{*}}(x) d x \leq C \sum_{i=1}^{m} \int_{\mathbb{R}^{n}}\left|M_{\alpha, \phi} f(x)\right|^{q_{*}} \omega^{q_{*}}\left(A_{i} x\right) d x
$$

Now, for any $k \in \mathbb{N}$, we assume that the results hold for all $0 \leq j \leq k-1$, and let us see how to derive the case $k$. For $\omega^{r} \in A\left(p / r, q_{*} / r\right)$, by Remark 2.1, we know that $\omega \in A\left(p, q_{*}\right)$. Then

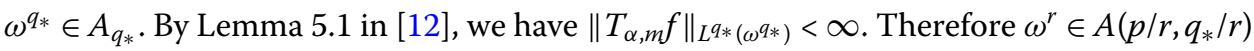
and $b \in L^{\infty}$, we have

$$
\left\|T_{\alpha, m, b}^{k} f\right\|_{L^{q_{*}\left(\omega^{q *}\right)}}=\left\|\sum_{j=0}^{k} c_{k, j} b^{k-j} T_{\alpha, m}\left(b^{j} f\right)\right\|_{L^{q *}\left(\omega^{q *}\right)}<\infty
$$

Besides, for $p<p_{l} \leq q_{*}, \omega \in A\left(p, q_{*}\right)$ implies $\omega \in A\left(p_{l}, q_{*}\right)$, and $1 / q_{*}=1 / p_{l}-(k-l) \beta / n$ implies $q_{*}=p_{k}$ when $l=k$. Then there exists $C>0$ such that

$$
\left\|M_{(k-l) \beta}\left(T_{\alpha, m, b}^{l} f\right)\right\|_{L^{q_{*}\left(\omega^{\left.q_{*}\right)}\right.}} \leq C\left\|T_{\alpha, m, b}^{l} f\right\|_{L^{p_{l}\left(\omega^{\left.p_{l}\right)}\right.}} .
$$

By the induction hypothesis, for $0 \leq l \leq k-1$ and $1 / p_{l}=1 / q_{j}-(l-j) \beta / n$, we get

$$
\left\|T_{\alpha, m, b}^{l} f\right\|_{L^{p_{l}\left(\omega^{\left.p_{l}\right)}\right.}} \leq C\|b\|_{\dot{\Lambda}_{\beta}}^{l} \sum_{i=1}^{m} \sum_{j=0}^{l}\left(\int_{\mathbb{R}^{n}}\left|M_{\alpha+j \beta, \phi} f(x)\right|^{q_{j}} \omega^{q_{j}}\left(A_{i} x\right) d x\right)^{1 / q_{j}} .
$$

Since $1 / q_{*}=1 / p_{l}-(k-l) \beta / n$ and $1 / p_{l}=1 / q_{j}-(l-j) \beta / n$, which implies $p_{l}=q_{j}$ when $l=j$, we have

$$
\begin{aligned}
& \left\|T_{\alpha, m, b}^{k} f\right\|_{\left.L^{q_{*}(\omega} q_{*}\right)} \\
& \leq\left(\int_{\mathbb{R}^{n}}\left|M\left(T_{\alpha, m, b}^{k} f\right)^{\delta}(x)\right|^{q_{*} / \delta} \omega^{q_{*}}(x) d x\right)^{1 / q_{*}} \\
& \leq\left(\int_{\mathbb{R}^{n}}\left|M_{\delta}^{\sharp}\left(T_{\alpha, m, b}^{k} f\right)(x)\right|^{q_{*}} \omega^{q_{*}}(x) d x\right)^{1 / q_{*}} \\
& \leq C \sum_{l=0}^{k-1}\|b\|_{\dot{\Lambda}_{\beta}}^{k-l}\left\|M_{(k-l) \beta}\left(T_{\alpha, m, b}^{l} f\right)(x)\right\|_{L^{q_{*}}\left(\omega^{\left.q_{*}\right)}\right.} \\
& \quad+C\|b\|_{\dot{\Lambda}_{\beta}}^{k} \sum_{i=1}^{m}\left(\int_{\mathbb{R}^{n}}\left(M_{\alpha+k \beta, \phi} f\left(A_{i}^{-1} x\right)\right)^{q_{*}} \omega^{q_{*}}(x) d x\right)^{1 / q_{*}} \\
& \leq C\|b\|_{\dot{\Lambda}_{\beta}}^{k} \sum_{i=1}^{m} \sum_{l=0}^{k-1} \sum_{j=0}^{l}\left(\int_{\mathbb{R}^{n}}\left|M_{\alpha+j \beta, \phi} f(x)\right|^{q_{j}} \omega^{q_{j}}\left(A_{i} x\right) d x\right)^{1 / q_{j}} \\
& \quad+C\|b\|_{\dot{\Lambda}_{\beta}}^{k} \sum_{i=1}^{m}\left(\int_{\mathbb{R}^{n}}\left(M_{\alpha+k \beta, \phi} f\left(A_{i}^{-1} x\right)\right)^{q_{*}} \omega^{q_{*}}(x) d x\right)^{1 / q_{*}}
\end{aligned}
$$




$$
\begin{aligned}
\leq & C\|b\|_{\dot{\Lambda}_{\beta}}^{k} \sum_{i=1}^{m} \sum_{j=0}^{k-1}\left(\int_{\mathbb{R}^{n}}\left|M_{\alpha+j \beta, \phi} f(x)\right|^{q_{j}} \omega^{q_{j}}\left(A_{i} x\right) d x\right)^{1 / q_{j}} \\
& +C\|b\|_{\dot{A}_{\beta}}^{k} \sum_{i=1}^{m}\left(\int_{\mathbb{R}^{n}}\left(M_{\alpha+k \beta, \phi} f(x)\right)^{q_{*}} \omega^{q_{*}}\left(A_{i} x\right) d x\right)^{1 / q_{*}} \\
\leq & C\|b\|_{\dot{\Lambda}_{\beta}}^{k} \sum_{i=1}^{m} \sum_{l=0}^{k-1}\left(\int_{\mathbb{R}^{n}}\left|M_{\alpha+l \beta, \phi} f(x)\right|^{p_{l}} \omega^{p_{l}}\left(A_{i} x\right) d x\right)^{1 / p_{l}} \\
& +C\|b\|_{\dot{\Lambda}_{\beta}}^{k} \sum_{i=1}^{m}\left(\int_{\mathbb{R}^{n}}\left(M_{\alpha+k \beta, \phi} f(x)\right)^{p_{k}} \omega^{p_{k}}\left(A_{i} x\right) d x\right)^{1 / p_{k}} \\
\leq & C\|b\|_{\dot{\Lambda}_{\beta}}^{k} \sum_{i=1}^{m} \sum_{l=0}^{k}\left(\int_{\mathbb{R}^{n}}\left(M_{\alpha+l \beta, \phi} f(x)\right)^{p_{l}} \omega^{p_{l}}\left(A_{i} x\right) d x\right)^{1 / p_{l}} .
\end{aligned}
$$

Namely,

$$
\left\|T_{\alpha, m, b}^{k} f\right\|_{L^{q_{*}\left(\omega q^{*}\right)}} \leq C\|b\|_{\dot{\Lambda}_{\beta}}^{k} \sum_{i=1}^{m} \sum_{l=0}^{k}\left(\int_{\mathbb{R}^{n}}\left(M_{\alpha+l \beta, \phi} f(x)\right)^{p_{l}} \omega^{p_{l}}\left(A_{i} x\right) d x\right)^{1 / p_{l}} .
$$

For the general case, if $b \in \dot{\Lambda}_{\beta}$, for any $N \in \mathbb{N}$, we define $b_{N}=b \chi_{\{x:-N<b(x)<N\}}+$ $N \chi_{\{x: b(x) \geq N\}}-N \chi_{\{x: b(x) \leq-N\}}$, then $\left\|b_{N}\right\|_{\dot{\Lambda}_{\beta}} \leq c\|b\|_{\dot{\Lambda}_{\beta}}$. Using convergence theorems, for details see [21], we conclude that (8) holds for any $b \in \dot{\Lambda}_{\beta}$ and $\omega^{r} \in A\left(p / r, q_{*} / r\right)$. Thus, as mentioned, using the extrapolation results obtained in [20], (3) holds for all $0<q<\infty$, $b \in \dot{\Lambda}_{\beta}$, and $\omega^{r} \in A(p / r, q / r)$.

If $\omega$ satisfies (2), we have

$$
\begin{aligned}
\left\|T_{\alpha, m, b}^{k} f\right\|_{L^{q}\left(\omega^{q}\right)} & \leq C\|b\|_{\dot{\Lambda}_{\beta}}^{k} \sum_{i=1}^{m} \sum_{l=0}^{k}\left(\int_{\mathbb{R}^{n}}\left(M_{\alpha+l \beta, \phi} f(x)\right)^{p_{l}} \omega^{p_{l}}\left(A_{i} x\right) d x\right)^{1 / p_{l}} \\
& \leq C\|b\|_{\dot{\Lambda}_{\beta}}^{k} \sum_{l=0}^{k}\left\|M_{\alpha+l \beta, \phi} f\right\|_{L^{p_{l}\left(\omega^{\left.p^{l}\right)}\right)}}
\end{aligned}
$$

which completes the proof of Theorem 1.2.

\section{The weighted inequalities of commutators}

This section is concerned with the proofs of Theorems 1.3 and 1.4. For the proof of Theorem 1.3, we need the Coifman inequality (3) and the boundedness of the maximal operator, given in [22] (see Theorem 2.6). Let us begin with the following previous result.

Theorem 4.1 ([22]) Let $0 \leq \alpha<n, \omega$ be a weight, $1 \leq r<p<n /(\alpha+k \beta)$, and $1 / q=1 / p-$ $(\alpha+k \beta) / n$. Let $\phi$ be a Young function such that $\phi^{1+\frac{\rho(\alpha+k \beta)}{n-(\alpha+k \beta)}} \in B_{\frac{\rho n}{n-(\alpha+k \beta)}}$ for every $\rho>r(n-$ $(\alpha+k \beta)) /(n-(\alpha+k \beta) r)$, and let $\eta$ be a Young function such that $\eta^{-1}(t) t^{(\alpha+k \beta) / n} \lesssim \phi^{-1}(t)$ for every $t>0$. If $\omega^{r} \in A(p / r, q / r)$, then $M_{\alpha+k \beta, \phi}$ is bounded from $L^{p}\left(\omega^{p}\right)$ to $L^{q}\left(\omega^{q}\right)$.

Now we are in a position to prove Theorem 1.3.

Proof of Theorem 1.3 For $1 / q=1 / p_{l}-(k-l) \beta / n$ and $1 / q=1 / p-(\alpha+k \beta) / n$, we can know $p<p_{l}<q$. Then, for $\omega^{r} \in A(p / r, q / r)$, by Remark 2.1, we can get $\omega^{r} \in A\left(p / r, p_{l} / r\right)$ and 
$\omega \in A(p, q)$; moreover, $\omega \in A\left(p_{l}, q\right)$. Therefore, from Theorem 4.1, we know that $M_{\alpha+l \beta, \phi}$ is bounded from $L^{p}\left(\omega^{p}\right)$ into $L^{p_{l}}\left(\omega^{p_{l}}\right)$. Then, by Theorem 1.2 and $\omega$ satisfies (2), we have

$$
\left\|T_{\alpha, m, b}^{k} f\right\|_{L^{q}\left(\omega^{q}\right)} \leq C\|b\|_{\dot{\Lambda}_{\beta}}^{k} \sum_{l=0}^{k}\left\|M_{\alpha+l \beta, \phi} f\right\|_{L^{p l}\left(\omega^{p} l\right)} \leq C\|b\|_{\dot{\Lambda}_{\beta}}^{k}\|f\|_{L^{p}\left(\omega^{p}\right)} .
$$

This proves the conclusion of Theorem 1.3.

Finally, we give the proof of Theorem 1.4.

Proof of Theorem 1.4 Following the ideas in [21], for $\omega^{r} \in A\left(\frac{n}{(\alpha+k \beta) r}, \infty\right)$, we know

$$
\left\|\omega M_{\alpha+k \beta, r} f\right\|_{\infty} \leq\|f \omega\|_{n /(\alpha+k \beta)} .
$$

Note that $\omega^{r} \in A\left(\frac{n}{(\alpha+k \beta) r}, \infty\right)$ implies $\omega^{r} \in A\left(\frac{n}{(k-l) \beta r}, \infty\right)$, we have

$$
\left\|\omega M_{(k-l) \beta, r} T_{\alpha, m, b}^{l} f\right\|_{\infty} \leq\left\|T_{\alpha, m, b}^{l} f \omega\right\|_{n /(k-l) \beta} .
$$

Now, by (9), (10), (2), Proposition 2.1, Theorems 1.1 and 1.3, we get

$$
\begin{aligned}
& \left\|T_{\alpha, m, b}^{k} f \mid\right\|_{\omega} \simeq\left\|\omega M^{\sharp} T_{\alpha, m, b}^{k} f\right\|_{\infty} \\
& \leq C \sum_{l=0}^{k-1}\|b\|_{\dot{\Lambda}_{\beta}}^{k-l}\left\|\omega M_{(k-l) \beta}\left(T_{\alpha, m, b}^{l} f\right)\right\|_{\infty} \\
& +C\|b\|_{\dot{\Lambda}_{\beta}}^{k} \sum_{i=1}^{m}\left\|\omega M_{\alpha+k \beta, \phi} f\left(A_{i}^{-1} \cdot\right)\right\|_{\infty} \\
& \leq C \kappa_{r_{1}} \sum_{l=0}^{k-1}\|b\|_{\dot{\Lambda}_{\beta}}^{k-l}\left\|\omega M_{(k-l) \beta, r}\left(T_{\alpha, m, b}^{l} f\right)\right\|_{\infty} \\
& +C \kappa_{r_{2}}\|b\|_{\dot{\Lambda}_{\beta}}^{k} \sum_{i=1}^{m}\left\|\omega M_{\alpha+k \beta, r} f\left(A_{i}^{-1} \cdot\right)\right\|_{\infty} \\
& \leq C \kappa_{r_{1}} \sum_{l=0}^{k-1}\|b\|_{\dot{\Lambda}_{\beta}}^{k-l}\left\|\omega T_{\alpha, m, b}^{l} f\right\|_{n /(k-l) \beta} \\
& +C \kappa_{r_{2}}\|b\|_{\dot{\Lambda}_{\beta}}^{k} \sum_{i=1}^{m}\left\|\omega f\left(A_{i}^{-1} \cdot\right)\right\|_{n /(\alpha+k \beta)} \\
& \leq C \kappa_{r_{1}} \sum_{l=0}^{k-1}\|b\|_{\dot{\Lambda}_{\beta}}^{k-l}\|b\|_{\dot{\Lambda}_{\beta}}^{l}\|\omega f\|_{n /(\alpha+k \beta)} \\
& +C \kappa_{r_{2}}\|b\|_{\dot{\Lambda}_{\beta}}^{k} \sum_{i=1}^{m}\left\|\omega f\left(A_{i}^{-1} \cdot\right)\right\|_{n /(\alpha+k \beta)} \\
& \leq C \kappa_{r_{1}}\|b\|_{\dot{\Lambda}_{\beta}}^{k}\|\omega f\|_{n /(\alpha+k \beta)} \\
& +C \kappa_{r_{2}}\|b\|_{\dot{\Lambda}_{\beta}}^{k} \sum_{i=1}^{m}\left\|\omega\left(A_{i} \cdot\right) f\right\|_{n /(\alpha+k \beta)}
\end{aligned}
$$




$$
\leq C\|b\|_{\dot{\Lambda}_{\beta}}^{k}\|f \omega\|_{L^{n / \alpha+k \beta}}
$$

This completes the proof of Theorem 1.4.

\section{Acknowledgements}

The authors thank the referees cordially for their valuable suggestions and comments.

\section{Funding}

This project is supported by the National Natural Science Foundation of China (Grant Nos. 11771358, 11871101).

\section{Competing interests}

The authors declare that they have no competing interests

\section{Authors' contributions}

All authors contributed equally and significantly in writing this paper. All authors read and approved the final manuscript.

\section{Publisher's Note}

Springer Nature remains neutral with regard to jurisdictional claims in published maps and institutional affiliations.

Received: 12 February 2019 Accepted: 25 July 2019 Published online: 06 August 2019

\section{References}

1. Ferreyra, E.V., Flores, G.J.: Weighted estimates for integral operators on local BMO type spaces. Math. Nachr. 288(8-9), 905-916 (2015)

2. Godoy, T., Saal, L., Urciuolo, M.: About certain singular kernels $k(x, y)=k_{1}(x-y) k_{2}(x+y)$. Math. Scand. 74(1), 98-110 (1994)

3. Godoy, T., Urciuolo, M.: About the $L^{p}$-boundedness of integral operators with kernels of the form $k_{1}(x-y) k_{2}(x+y)$. Math. Scand. 76(1), 84-92 (1996)

4. Godoy, T., Urciuolo, M.: On certain integral operators of fractional type. Acta Math. Hung. 82(1-2), $99-105$ (1999)

5. Riveros, M.S., Urciuolo, M.: Weighted inequalities for fractional type operators with some homogeneous kernels. Acta Math. Sin. Engl. Ser. 29(3), 449-460 (2013)

6. Riveros, M.S., Urciuolo, M.: Weighted inequalities for some integral operators with rough kernels. Open Math. 12(4), 636-647 (2014)

7. Rocha, P.: A remark on certain integral operators of fractional type (2017) arXiv:1703.03287

8. Rocha, P., Urciuolo, M.: About integral operators of fractional type on variable $L^{p}$ spaces. Georgian Math. J. 20(4), $805-816(2013)$

9. Urciuolo, M.: Weighted inequalities for integral operators with almost homogeneous kernels. Georgian Math. J. 13(1), 183-191 (2006)

10. Urciuolo, M., Vallejos, $L .: L^{p}(\cdot)-L^{q}(\cdot)$ boundedness of some integral operators with extrapolation techniques. Georgian Math. J. (2019, to appear). https://doi.org/10.1515/gmj-2018-0066

11. Riveros, M.S., Urciuolo, M.: Weighted inequalities for integral operators with some homogeneous kernels. Czechoslov. Math. J. 55(2), 423-432 (2005)

12. Ibañez-Firnkorn, G.H., Riveros, M.S.: Certain fractional type operators with Hörmander conditions. Ann. Acad. Sci. Fenn., Math. 43(2), 913-929 (2018)

13. Bernardis, A.L., Lorente, M., Riveros, M.S.: Weighted inequalities for fractional integral operators with kernel satisfying Hörmander type conditions. Math. Inequal. Appl. 14(4), 881-895 (2011)

14. Gallo, A.L., Ibañez-Firnkorn, G.H., Riveros, M.S.: Hörmander's conditions for vector-valued kernels of singular integrals and its commutators (2017) arXiv:1706.08357

15. Ibañez-Firnkorn, G.H., Riveros, M.S.: Commutators of certain fractional type operators with Hörmander condition, one-weighted and two-weighted inequalities (2018) arXiv:1804.10095

16. O'Neil, R.: Fractional integration in Orlicz spaces I. Trans. Am. Math. Soc. 115, 300-328 (1965)

17. Rao, M., Ren, Z.: Theory of Orlicz Spaces. Monographs and Textbooks in Pure and Applied Mathematics, vol. 146 (1991)

18. Cruz-Uribe, D., Moen, K.: A fractional Muckenhoupt-Wheeden theorem and its consequences (2013) arXiv:1303.3424

19. Caldarelli, M., Lerner, A., Ombrosi, S.: On a counterexample related to weighted weak type estimates for singular integrals. Proc. Am. Math. Soc. 145(7), 3005-3012 (2017)

20. Cruz-Uribe, D., Martell, J.M., Pérez, C.: Extrapolation from $A_{\infty}$ weights and applications. J. Funct. Anal. 213(2), $412-439$ (2004)

21. Lorente, M., Martell, J.M., Riveros, M.S., Torre, L.: Generalized Hörmander's conditions, commutators and weights J. Math. Anal. Appl. 342(2), 1399-1425 (2008)

22. Bernardis, A.L., Dalmasso, E., Pradolini, G.: Generalized maximal functions and related operators on weighted Musielak-Orlicz spaces. Ann. Acad. Sci. Fenn., Math. 39(1), 23-50 (2014) 